\title{
KEPLER-63b: A GIANT PLANET IN A POLAR ORBIT AROUND A YOUNG SUN-LIKE STAR
}

\author{
Roberto Sanchis-Ojeda ${ }^{1}$, Joshua N. Winn ${ }^{1}$, Geoffrey W. Marcy ${ }^{2}$, Andrew W. Howard ${ }^{3}$, Howard Isaacson ${ }^{2}$, \\ John Asher Johnson ${ }^{4,15}$, Guillermo Torres ${ }^{5}$, Simon Albrecht $^{1}$, Tiago L. Campante ${ }^{6}$, William J. Chaplin ${ }^{6}$, \\ Guy R. Davies ${ }^{6}$, Mikkel N. Lund ${ }^{7}$, Joshua A. Carter ${ }^{5}$, Rebekah I. Dawson ${ }^{5}$, Lars A. Buchhave ${ }^{8,9}$, \\ Mark E. Everett ${ }^{10,16}$, Debra A. Fischer ${ }^{11}$, John C. Geary ${ }^{5}$, Ronald L. Gilliland ${ }^{12}$, \\ Elliott P. Horch ${ }^{13,16,17}$, Steve B. Howell ${ }^{14,16}$, and David W. Latham ${ }^{5}$ \\ ${ }^{1}$ Department of Physics, and Kavli Institute for Astrophysics and Space Research, Massachusetts Institute of Technology, Cambridge, MA 02139, USA \\ 2 Astronomy Department, University of California, Berkeley, CA 94720, USA \\ ${ }^{3}$ Institute for Astronomy, University of Hawaii, 2680 Woodlawn Drive, Honolulu, HI 96822, USA \\ ${ }^{4}$ Department of Astronomy, California Institute of Technology, 1200 E. California Blvd., Pasadena, CA 91125, USA \\ ${ }^{5}$ Harvard-Smithsonian Center for Astrophysics, Cambridge, MA 02138, USA \\ ${ }^{6}$ School of Physics and Astronomy, University of Birmingham, Edgbaston, Birmingham B15 2TT, UK \\ ${ }^{7}$ Stellar Astrophysics Centre (SAC), Department of Physics and Astronomy, Aarhus University, Ny Munkegade 120, DK-8000 Aarhus C, Denmark \\ ${ }^{8}$ Niels Bohr Institute, University of Copenhagen, Juliane Maries vej 30, DK-2100 Copenhagen, Denmark \\ ${ }^{9}$ Centre for Star \& Planet Formation, Natural History Museum of Denmark, University of Copenhagen, Øster Voldgade 5-7, DK-1350 Copenhagen, Denmark \\ ${ }^{10}$ National Optical Astronomy Observatory, 950 N. Cherry Ave, Tucson, AZ 85719, USA \\ ${ }^{11}$ Astronomy Department, Yale University, New Haven, CT, USA \\ ${ }^{12}$ Center for Exoplanets and Habitable Worlds, The Pennsylvania State University, 525 Davey Lab, University Park, PA 16802, USA \\ ${ }^{13}$ Southern Connecticut State University, New Haven, CT 06515, USA \\ ${ }^{14}$ NASA Ames Research Center, Moffett Field, CA 94035, USA \\ Received 2013 March 23; accepted 2013 August 4; published 2013 September 4
}

\begin{abstract}
We present the discovery and characterization of a giant planet orbiting the young Sun-like star Kepler-63 (KOI-63, $m_{\mathrm{Kp}}=11.6, T_{\text {eff }}=5576 \mathrm{~K}, M_{\star}=0.98 M_{\odot}$ ). The planet transits every 9.43 days, with apparent depth variations and brightening anomalies caused by large starspots. The planet's radius is $6.1 \pm 0.2 R_{\oplus}$, based on the transit light curve and the estimated stellar parameters. The planet's mass could not be measured with the existing radial-velocity data, due to the high level of stellar activity, but if we assume a circular orbit, then we can place a rough upper bound of $120 M_{\oplus}(3 \sigma)$. The host star has a high obliquity $\left(\psi=104^{\circ}\right)$, based on the Rossiter-McLaughlin effect and an analysis of starspot-crossing events. This result is valuable because almost all previous obliquity measurements are for stars with more massive planets and shorter-period orbits. In addition, the polar orbit of the planet combined with an analysis of spot-crossing events reveals a large and persistent polar starspot. Such spots have previously been inferred using Doppler tomography, and predicted in simulations of magnetic activity of young Sun-like stars.
\end{abstract}

Key words: planetary systems - stars: activity - stars: individual (Kepler-63) - stars: rotation - starspots

Online-only material: color figures

\section{INTRODUCTION}

There are good reasons why planet hunters try to avoid chromospherically active stars. For those who use the radial-velocity (RV) technique, starspots and plages distort the absorption lines, inducing RV signals that can be similar to those of planets (see, for example, Lovis et al. 2011). A good example of these complications is CoRoT-7 (Léger et al. 2009), for which different authors have measured different planet masses based on the same RV data, due to the strong activity of the host star (Queloz et al. 2009; Pont et al. 2011; Ferraz-Mello et al. 2011; Hatzes et al. 2011).

Starspots can also be a source of noise in the transit technique. Starspots are carried around the star by rotation, inducing flux variations that could be hard to detect from groundbased telescopes. When they go unnoticed, they can bias the determination of the transit parameters (Czesla et al. 2009; Carter et al. 2011). In addition, when the planet crosses over a dark starspot, it temporarily blocks less light than expected, causing a brightening anomaly (Silva 2003). These can be an

\footnotetext{
${ }^{15}$ Sloan Fellow; Packard Fellow.

${ }^{16}$ Visiting Astronomer Kitt Peak National Observatory.

${ }^{17}$ Adjunct Astronomer, Lowell Observatory.
}

additional source of error, or be confused with transits of other bodies in the system (Pont et al. 2007; Rabus et al. 2009).

Space-based transit surveys have the potential to overcome these problems, thanks to their high photometric precision and nearly continuous time coverage. The data from these surveys provide the opportunity to study the general activity levels of thousands of stars (Basri et al. 2011) as well as spot evolution and magnetic cycles of individual systems (Bonomo \& Lanza 2012). With hundreds or even thousands of transiting objects detected to date, spot-crossing events are more readily observed. They bear information about the sizes, temperatures, and positions of the spots (Silva 2003), as well as the stellar rotation period (Silva-Valio 2008; Dittmann et al. 2009).

Spot-crossing events can also provide information about the architecture of exoplanetary systems. Measurements of the angle between the spin axis of the star and the orbital plane of the planet (known as the obliquity) can help test theories of formation and evolution of these systems (Queloz et al. 2000; Winn et al. 2005). Most of the obliquity measurements to date have been based on the Rossiter-McLaughlin (RM) effect, a spectroscopic effect observed during transits (see, e.g., the recent compilation by Albrecht et al. 2012). One can also test whether a transit-hosting star has a high obliquity using asteroseismology (Gizon \& Solanki 2003; Chaplin et al. 2013), 
the combination of $v \sin i_{\star}$, stellar radius, and stellar rotation period (Schlaufman 2010; Hirano et al. 2012), or starspotcrossing events (Sanchis-Ojeda et al. 2011; Nutzman et al. 2011; Désert et al. 2011; Tregloan-Reed et al. 2013).

The basic idea behind using starspot-crossing events to measure the obliquity is that when the obliquity is low, any such events are expected to recur in consecutive transits. This is because in such cases the trajectory of the spot is parallel to the trajectory of the planet across the stellar disk; when the planet transits again, the spot is likely to have remained on the transit chord and a spot-crossing event will occur at a later phase of the transit. In contrast, the rotation of a highly oblique star would carry the spot away from the transit chord, and the anomalies would not recur in consecutive transits. For a more detailed explanation and recent elaborations of this technique, we refer the reader to Sanchis-Ojeda et al. (2013). One interesting feature of the starspot-crossing technique is that in the case of a highly misaligned system, the planet may transit across a wide range of stellar latitudes (Deming et al. 2011; Sanchis-Ojeda \& Winn 2011). In these systems, we have the rare opportunity to measure the latitudes of starspots and their evolution in time.

In this paper, we present Kepler-63b, a new transiting planet discovered with the Kepler space telescope. The paper is organized as follows. Section 2 describes the Kepler observations and other follow-up observations necessary to confirm the planetary nature of Kepler-63b. Section 3 describes the effort to characterize the age, radius, and mass of the Kepler-63 host star. Section 4 explains how we characterized the planet in the presence of large and dark starspots. Section 5 demonstrates that Kepler-63 has a large obliquity, using the RM effect. Section 6 confirms the high obliquity of the system using spot-crossing events. Section 7 summarizes what we have learned about the starspots on Kepler-63, including their latitudes. The paper finishes with a discussion of the results in the context of current theories for stellar activity and planetary systems.

\section{OBSERVATIONS}

\subsection{Kepler Observations}

For more than four years, the Kepler space telescope monitored approximately 150,000 stars in the constellations of Cygnus and Lyra (Koch et al. 2010; Borucki et al. 2010). The observations consisted of a series of $6 \mathrm{~s}$ exposures that were combined into final images with an effective exposure time of 1 minute (short-cadence mode; Gilliland et al. 2010) or 29.4 minutes (long-cadence mode). The target star Kepler-63 was identified in the Kepler Input Catalog (Brown et al. 2011) as KIC 11554435 (also 2MASS J19165428+4932535) with $\mathrm{Ke}$ pler magnitude 11.58 and $V=12.02$. Because of the relative brightness of the star, and the high signal-to-noise ratio $(\mathrm{S} / \mathrm{N})$ of the flux dips, it was identified as a transiting-planet candidate early in the mission and designated Kepler Object of Interest (KOI) number 63. For this study we used long-cadence observations from quarters 1-12, spanning nearly three years (2009 May 13 through 2012 March 28). Short-cadence observations were also used whenever available (quarters 3-12, as well as one month in quarter 2).

The Kepler pipeline provides data with time stamps expressed in barycentric Julian days in the TDB (Barycentric Dynamical Time) system. Two sets of fluxes are provided: simple aperture photometry, which is known to be affected by several instrumental artifacts (Jenkins et al. 2010); and fluxes that have been corrected with an algorithm called PDC-MAP that attempts to remove the artifacts while preserving astrophysical sources of variability (Stumpe et al. 2012; Smith et al. 2012). For this study we used the PDC-MAP time series. Because of the large pixel scale (4 arcsec) of Kepler's detectors, it is always important to consider the possibility that the reported fluxes include contributions from neighboring stars ("blends"). The time series of the measured coordinates of the source of light are useful diagnostics. For Kepler-63, we used the coordinates of the center of light based on PSF-fitting (PSF_CENTR) which were provided by the Kepler pipeline (see 4.1).

\subsection{Spectroscopic Observations}

We used the spectroscopic observations gathered by the Kepler Follow-up Program. One spectrum was obtained with fiber-fed Tillinghast Reflector Échelle Spectrograph (TRES) on the $1.5 \mathrm{~m}$ Tillinghast Reflector at the Fred Lawrence Whipple Observatory on Mt. Hopkins, Arizona, with a resolution of 44,000. The observation took place on 2009 June 13 with an exposure time of 24 minutes, giving an S/N of 64 in the $\mathrm{Mg}$ I b order. Another spectrum was taken with the HIRES spectrograph (Vogt et al. 1994) on the $10 \mathrm{~m}$ Keck I telescope at Mauna Kea, Hawaii, with a resolution of 48,000. The observation took place on 2009 August 1 with an exposure time of 20 minutes, giving an $\mathrm{S} / \mathrm{N}$ of 250 . Three more spectra were taken with the FIber-fed Échelle Spectrograph (FIES) on the $2.5 \mathrm{~m}$ Nordic Optical Telescope on La Palma, Spain (Djupvik \& Andersen 2010), with a resolution of 46,000. The observations took place on 2010 June 2, 5, and 6, with a typical exposure time of 20 minutes, giving an $\mathrm{S} / \mathrm{N}$ of about 80 .

We conducted additional observations to try to measure the RV signal induced by the planet on the star, and also to detect the RM effect. We used HIRES on the Keck I 10 m telescope to obtain seven spectra during the two weeks before the transit of 2011 August 20/21. Then, during the night of the transit, we obtained 30 spectra with a typical exposure time of 10 minutes, starting $3 \mathrm{hr}$ before the transit and finishing $3 \mathrm{hr}$ afterward. We determined relative RVs in the usual way for HIRES, by analyzing the stellar spectra filtered through an iodine cell (wavelength range 500-600 nm). For the analysis, we used a modified version of the original code by Butler et al. (1996). Table 1 gives the RVs.

\subsection{Speckle Imaging}

High-resolution images are useful to establish which stars are contributing to the Kepler photometric signal. Speckle imaging was conducted on the night of 2010 September 17, using the twocolor DSSI speckle camera at the WIYN 3.5 m telescope on Kitt Peak, Arizona. The speckle camera simultaneously obtained images in two filters: $V(5460 / 400 \AA)$ and $R(6920 / 400 \AA)$. These data were processed to produce a final reconstructed speckle image for each filter. Details of the processing were presented by Howell et al. (2011). The speckle observations are sensitive to companions between 0.05 and 1.5 arcsec from Kepler-63. We found no companion star within this range of separations, and can place an upper limit on the brightness of such stars corresponding to $\Delta R=4-5$ mag and $\Delta V=3-5$ mag below the brightness of Kepler-63.

\section{STELLAR CHARACTERIZATION}

\subsection{Rotation Period and Age Estimate}

We identified Kepler-63 as an interesting target based on its high level of chromospheric activity, and the high $\mathrm{S} / \mathrm{N}$ with 
Table 1

Relative Radial Velocity Measurements of Kepler-63

\begin{tabular}{|c|c|c|}
\hline $\mathrm{BJD}_{\mathrm{TDB}}$ & $\begin{array}{c}\mathrm{RV} \\
\left(\mathrm{m} \mathrm{s}^{-1}\right)\end{array}$ & $\begin{array}{c}\text { Unc. } \\
\left(\mathrm{m} \mathrm{s}^{-1}\right)\end{array}$ \\
\hline 2455782.054444 & -19.6 & 3.3 \\
\hline 2455782.937924 & 31.3 & 3.0 \\
\hline 2455787.771381 & -26.7 & 3.0 \\
\hline 2455788.809546 & -5.6 & 3.2 \\
\hline 2455789.858820 & -7.5 & 3.1 \\
\hline 2455790.807157 & -23.2 & 2.8 \\
\hline 2455792.775137 & -37.4 & 2.7 \\
\hline 2455793.743264 & 4.2 & 2.2 \\
\hline 2455793.750173 & -4.9 & 2.1 \\
\hline 2455793.757280 & 0.6 & 2.1 \\
\hline 2455793.791469 & -2.5 & 2.2 \\
\hline 2455793.798830 & 1.3 & 2.2 \\
\hline 2455793.806157 & -2.7 & 2.2 \\
\hline 2455793.813541 & -2.3 & 2.0 \\
\hline 2455793.821018 & -1.4 & 2.3 \\
\hline 2455793.828321 & 7.8 & 2.2 \\
\hline 2455793.835717 & 2.2 & 2.1 \\
\hline 2455793.843124 & 2.7 & 2.2 \\
\hline 2455793.850578 & 13.2 & 2.1 \\
\hline 2455793.857834 & 16.6 & 2.6 \\
\hline 2455793.865369 & 11.6 & 2.4 \\
\hline 2455793.872800 & 18.8 & 2.4 \\
\hline 2455793.880404 & 20.8 & 2.4 \\
\hline 2455793.887626 & 9.9 & 2.3 \\
\hline 2455793.895195 & 7.3 & 2.2 \\
\hline 2455793.902278 & 15.1 & 2.8 \\
\hline 2455793.912105 & 0.3 & 3.0 \\
\hline 2455793.919987 & 12.5 & 3.3 \\
\hline 2455793.927382 & 5.9 & 2.6 \\
\hline 2455793.934894 & -7.0 & 2.7 \\
\hline 2455793.942232 & 3.8 & 2.8 \\
\hline 2455793.949546 & -7.3 & 2.9 \\
\hline 2455793.957266 & -3.3 & 3.3 \\
\hline 2455793.964546 & -19.0 & 3.1 \\
\hline 2455793.972081 & 0.7 & 3.1 \\
\hline 2455793.979569 & -6.7 & 3.1 \\
\hline 2455794.010807 & -10.1 & 2.9 \\
\hline
\end{tabular}

Notes. RVs were measured relative to an arbitrary template spectrum; only the differences are significant. Column 3 gives the internally estimated measurement uncertainty which does not account for any "stellar jitter."

which individual transits are detected. The high level of activity is evident from the quasi-periodic stellar flux variations. To study these variations, we used the published transit ephemeris (Batalha et al. 2013) to remove the transit signals. To reject outliers, we clipped those data points more than $3 \sigma$ away from the median flux over the surrounding $10 \mathrm{hr}$ interval. Since the star's light fell on a different CCD during each of the four Kepler observing seasons, we had to make a choice for the normalization of each quarterly time series. We chose to divide each quarterly time series by the mean flux in that quarter, since it seemed to be the easiest way to avoid large flux discontinuities between quarters.

The upper panel of Figure 1 shows the relative flux time series. The short-term variability on a scale of a few days is presumably caused by spots being carried around by the rotation of the star. There is also long-term variability, probably reflecting spot evolution. The peak-to-peak variability reaches a maximum value near $4 \%$. There are also intervals with much lower variability. A Lomb-Scargle periodogram (Scargle 1982) shows

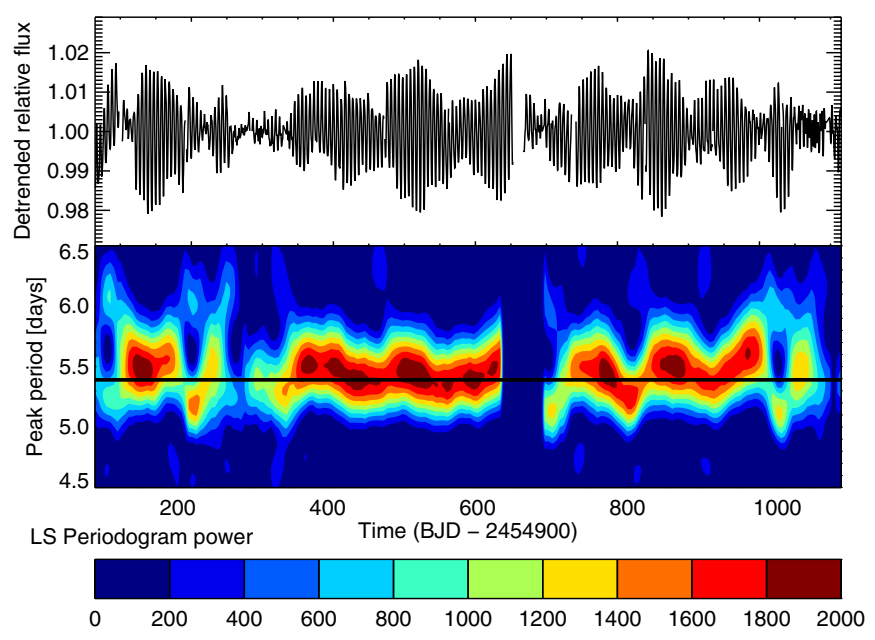

Figure 1. Determination of the stellar rotation period from Kepler photometry. Top: time series used to estimate the rotation period, after removing the transit signals. The short-term variability is presumably from spots being carried by the stellar rotation. The longer-term variations may be caused by spot evolution. Bottom: running periodogram. The red colors represent high power, and the black line is where $P_{\text {rot }} / P_{\text {orb }}=4 / 7$.

(A color version of this figure is available in the online journal.)

a strong peak at 5.4 days, with a full width at half-maximum of 0.014 days. Sometimes the highest peak in a periodogram actually represents a harmonic of the true period, but in this case there is no significant signal at twice the candidate period and there is a (weaker) signal at half the candidate period, supporting our identification. Therefore, we interpret the strongest peak as the rotation period, and adopt $P_{\text {rot }}=5.401 \pm 0.014$ days.

This is nearly commensurate with the orbital period of Kepler-63b, with $P_{\text {rot }} / P_{\text {orb }}=(4.01 \pm 0.01) / 7$. Whether this relationship is caused by a physical process or is merely a coincidence, it has an important consequence for the interpretation of spot-crossing anomalies, as pointed out by Winn et al. (2010b) in the context of the HAT-P-11 system. The near-commensurability causes a "stroboscopic" effect in the pattern of anomalies, and may lead to the detection of recurrences even in the case of misaligned systems. For example, Désert et al. (2011) used the stroboscopic effect to boost the $\mathrm{S} / \mathrm{N}$ of the spot signals in the Kepler-17b system.

Other lower-power peaks are present in the periodogram, which could be a sign of differential rotation or spot evolution. We checked for any variability of the position of the highestpower peak by computing a running periodogram: for each time $t$ we calculated the Lomb-Scargle periodogram of a 50 day time interval centered on $t$. We performed these periodograms for a sequence of $t$ values spaced apart by 5 days. Since there are occasional gaps in the data collection, we only computed periodograms for those intervals for which the gaps constitute less than $20 \%$ of the interval. (Removing the transits only eliminates $2 \%$ of the data.) The results are shown in the lower part of Figure 1, with the red colors indicating higher periodogram power. The black line is where $P_{\text {rot }} / P_{\text {orb }}=4 / 7$. It seems that the highest peak was quite stable over the three years of observations.

Our spectroscopic analysis (see Section 2.2) shows that Kepler-63 is a Sun-like star. With such a short rotation period of 5.4 days, and assuming the star has not been spun up due to tidal or other interactions, Kepler-63 is likely to be relatively young. The Sun had a rotation period of 6 days at an age of approximately $300 \mathrm{Myr}$, based on the Skumanich (1972) law in which the rotation period grows as the square root of 
time. None of the Sun-like stars in the Hyades or Praesepe have rotation periods as short as 5.4 days (Irwin \& Bouvier 2009). Thus, Kepler-63 is likely younger than $650 \mathrm{Myr}$, which is the approximate age of these clusters. Schlaufman (2010) presented a convenient polynomial relationship between stellar mass, age, and rotation period; using this relationship, we find for Kepler-63 an age of $210 \pm 35 \mathrm{Myr}$. We also used the polynomial relationship given by Barnes (2007) and updated by Meibom et al. (2009); applying this relationship with $B-V=0.7$ for Kepler-63 gives an age of $210 \pm 45$ Myr.

Independent evidence for youth comes from a high observed level of chromospheric emission. The Mt. Wilson $S_{\mathrm{HK}}$ index, obtained from the Keck spectra, has an average value of 0.37 , from which we estimate a chromospheric flux ratio $\log R_{\mathrm{HK}}^{\prime}=$ -4.39. Using the correlation between chromospheric emission and rotation period presented by Noyes et al. (1984), and an estimated $B-V=0.7$ for this star, one would expect the star's rotation period to be 6.8 days, in good agreement with our photometrically-derived period. The relationship presented by Mamajek \& Hillenbrand (2008) between $\log R_{\mathrm{HK}}^{\prime}$ and stellar age gives in this case an age of $280 \mathrm{Myr}$.

\subsection{Stellar Dimensions}

At first, we determined the star's spectroscopic parameters by applying the spectral synthesis code Spectroscopy Made Easy (SME; Valenti \& Fischer 2005) to the Keck spectrum. The results were $T_{\text {eff }}=5698 \pm 44 \mathrm{~K},[\mathrm{Fe} / \mathrm{H}]=+0.26 \pm 0.04$, $v \sin i_{\star}=5.8 \pm 0.5 \mathrm{~km} \mathrm{~s}^{-1}$, and $\log g=4.64 \pm 0.06$. We then recognized that this value of $\log g$ is anomalously high for a young Sun-like star, which was otherwise suggested by the star's effective temperature and relatively short rotation period. One would instead expect log $g$ to be closer to the Solar value of 4.44. In fact, by consulting the Yonsei-Yale $\left(\mathrm{Y}^{2}\right)$ stellar evolution models (Yi et al. 2001), we found that the SME-based value of $\log g$ would place the star in a very unusual location on the theoretical H-R diagram, at a higher gravity than any of the isochrones for the nominal metallicity. This mismatch probably reflects the well known biases in spectroscopic determinations of $\log g$ (see, e.g., Torres et al. 2012).

To address this issue, we used an updated version of the Stellar Parameter Classification code (SPC; Buchhave et al. 2012) which determines the spectroscopic parameters subject to a prior constraint on the surface gravity. In this implementation, SPC is used to provide an initial guess at the effective temperature and metallicity for the star and select the $\mathrm{Y}^{2}$ evolutionary models that are compatible with this initial guess within fairly wide intervals $\left( \pm 250 \mathrm{~K}\right.$ in $T_{\text {eff }}, \pm 0.3 \mathrm{dex}$ in $\left.[\mathrm{m} / \mathrm{H}]^{18}\right)$. The selected evolutionary models then provide an interval of allowed surface gravities, which are used to construct a prior on the surface gravity for a second iteration of SPC. The final results, based on weighted averages of the results for all of the spectra (three FIES, two TRES, and one HIRES), are $T_{\text {eff }}=5576 \pm 50 \mathrm{~K}$, $[\mathrm{m} / \mathrm{H}]=0.05 \pm 0.08, \log g=4.52 \pm 0.10$, and $v \sin i_{\star}=$ $5.4 \pm 0.5 \mathrm{~km} \mathrm{~s}^{-1}$.

Finally, we used the $\mathrm{Y}^{2}$ models to determine the stellar dimensions, based not only on the spectroscopic parameters but also the rotation-based constraint on the stellar age (see Section 3.1). A comparison of our spectroscopic parameters with the $\mathrm{Y}^{2}$ models for ages between 100 and $600 \mathrm{Myr}$

\footnotetext{
18 Throughout this paper, the generic metallicity index $[\mathrm{m} / \mathrm{H}]$ computed with $\mathrm{SPC}$ in the $\mathrm{Mg}$ I b region will be considered as equivalent to the more commonly used $[\mathrm{Fe} / \mathrm{H}]$ index, as is usually the case for stars with near-solar composition.
}

suggests a surface gravity of $\log g=4.52 \pm 0.02$. Using the initial spectroscopic parameters, but with the smaller $0.02 \mathrm{dex}$ uncertainty on $\log g$, we then used the $\mathrm{Y}^{2}$ models to determine the stellar mass $\left(M_{\star}\right)$, radius $\left(R_{\star}\right)$, and mean density $\left(\rho_{\star}\right)$. We proceeded in a Monte Carlo fashion, randomly drawing 100,000 sets of temperature, metallicity, and surface gravity values from assumed Gaussian distributions of those quantities based on the above results, and inferring the stellar properties for each set. Our final results were obtained from the mode of the corresponding posterior distributions, and the (" $1 \sigma$ ") uncertainties from the $15.85 \%$ and $84.15 \%$ percentiles of the cumulative distributions. These values are reported in Table 2. Based on the absolute and apparent magnitudes, the distance to the system is $200 \pm 15$ pc.

\section{PLANET CHARACTERIZATION}

\subsection{Constraints on Blend Scenarios}

The speckle image of Kepler-63 (see Section 2.3) puts tight constraints on any background star that could be responsible for the transit signal. We also used the PSF-fitted image centroids provided with the Kepler data to further restrict the possibilities for background blend scenarios (Batalha et al. 2010; Bryson et al. 2013). Using a similar approach as Chaplin et al. (2013), we selected the long-cadence column and row centroids within a two hour interval centered on each transit, and used the surrounding three hours of data before and after the transits to correct for linear trends caused by pointing drifts and other instrumental effects. We phase-folded the centroid data and computed the mean differences between the row and column values inside and outside of the transits. The centroid shifts were $12 \pm 11 \mu$ pix in the column direction and $28 \pm 14 \mu$ pix in the row direction.

If the source of the transits were a background star situated at a distance $\Delta x$ from Kepler-63, the expected centroid shift would be approximately $d x=(\Delta x) \delta$, where $\delta$ is the transit depth. Adding both the row and column shift in quadrature, we obtain $d x=30 \pm 18 \mu$ pix, or $d x<84 \mu$ pix $(3 \sigma)$. The radius of confusion $r$, defined as the maximum angular separation of any hypothetical background source that could be responsible for the transit signal, can be obtained by dividing by the transit depth and multiplying by 4 arcsec pix ${ }^{-1}$, giving $r=0.084$ arcsec. The more sophisticated techniques described by Bryson et al. (2013) indicate that the source of photometric variability is offset from the bright target star by $0.02 \pm 0.02$ arcsec (based on the Data Validation Reports provided by the Kepler team). These results are compatible with Kepler-63 as the origin of the flux variations, and require any hypothetical background source of the variations to be aligned with Kepler-63 to within a small fraction of an arcsecond, an unlikely coincidence.

This type of analysis cannot exclude the possibility that the transit signal is caused by a planet orbiting a companion star that is gravitationally bound to the intended target star. However, the starspot anomalies that are detected in many transits show that the planet is orbiting a heavily spotted star. This star must be the main source of light in the aperture, because the $4 \%$ flux variations observed would be unphysically large if they actually represented the diluted variations of a fainter star. This possibility is also excluded by the good agreement between the temperature of the occulted spots and the size of the flux variations observed (see Section 7). We conclude that the transitlike signals do indeed arise from transits of a planet around the star Kepler-63. 
Table 2

System Parameters of Kepler-63

\begin{tabular}{|c|c|c|c|}
\hline Parameter & Value & $68.3 \%$ Conf. Limits & Note \\
\hline KIC number/KOI number & $11554435 / 63$ & & \\
\hline Kepler apparent magnitude & 11.582 & & \\
\hline Right ascension (J2000) & $19^{\mathrm{h}} 16^{\mathrm{m}} 54^{\mathrm{s}} .28$ & & \\
\hline Declination (J2000) & $+49^{\circ} 32^{\prime} 53^{\prime \prime} .52$ & & \\
\hline Stellar surface gravity, $\log \left(g\left(\mathrm{~cm} \mathrm{~s}^{-2}\right)\right)$ & 4.52 & \pm 0.02 & a \\
\hline Stellar effective temperature, $T_{\text {eff }}(\mathrm{K})$ & 5576 & \pm 50 & $\mathrm{~b}$ \\
\hline Stellar metallicity $[\mathrm{Fe} / \mathrm{H}]$ & 0.05 & \pm 0.08 & $\mathrm{~b}$ \\
\hline Stellar mass, $M_{\star}\left(M_{\odot}\right)$ & 0.984 & $-0.04,+0.035$ & $\mathrm{~b}$ \\
\hline Stellar radius, $R_{\star}\left(R_{\odot}\right)$ & 0.901 & $-0.022,+0.027$ & $\mathrm{~b}$ \\
\hline Stellar mean density, $\rho_{\star}\left(\rho_{\odot}\right)$ & 1.345 & $-0.083,+0.089$ & $\mathrm{~b}$ \\
\hline Stellar luminosity, $L_{\star}\left(L_{\odot}\right)$ & 0.696 & $-0.059,+0.076$ & $\mathrm{~b}$ \\
\hline Stellar rotation period (days) & 5.401 & \pm 0.014 & $\mathrm{c}$ \\
\hline Mt. Wilson chromospheric index $S_{\mathrm{HK}}$ & 0.37 & & $\mathrm{~d}$ \\
\hline Chromospheric flux ratio $\log R_{\mathrm{HK}}^{\prime}$ & -4.39 & & $\mathrm{~d}$ \\
\hline Distance from Earth $(\mathrm{pc})$ & 200 & \pm 15 & $\mathrm{~b}$ \\
\hline Reference epoch $\left(\mathrm{BJD}_{\mathrm{TDB}}\right)$ & 2455010.84307 & \pm 0.00005 & e \\
\hline Orbital period (days) & 9.4341505 & \pm 0.0000010 & $\mathrm{e}$ \\
\hline Planet-to-star radius ratio, $R_{\mathrm{p}} / R_{\star}$ & 0.0622 & \pm 0.0010 & $\mathrm{e}$ \\
\hline Transit impact parameter, $b$ & 0.732 & \pm 0.003 & $\mathrm{e}$ \\
\hline Scaled semimajor axis, $a / R_{\star}$ & 19.12 & \pm 0.08 & $\mathrm{e}$ \\
\hline Transit duration (1st to 4 th contact) (hr) & 2.903 & \pm 0.003 & $\mathrm{e}$ \\
\hline Transit duration (1.5 to 3.5$)(\mathrm{hr})$ & 2.557 & \pm 0.004 & $\mathrm{e}$ \\
\hline Transit ingress or egress duration (hr) & 0.346 & \pm 0.004 & $\mathrm{e}$ \\
\hline Linear limb-darkening coefficient, $u_{1}$ & 0.31 & \pm 0.04 & $\mathrm{e}$ \\
\hline Quadratic limb-darkening coefficient, $u_{2}$ & 0.354 & $-0.05,+0.07$ & $\mathrm{e}$ \\
\hline Orbital inclination, $i$ (deg) & 87.806 & $-0.019,+0.018$ & $\mathrm{e}$ \\
\hline Orbital eccentricity, $e$ & $<0.45(3 \sigma)$ & & $\mathrm{f}$ \\
\hline Orbital semimajor axis (AU) & 0.080 & \pm 0.002 & $\mathrm{~b}, \mathrm{e}$ \\
\hline Planet radius, $R_{p}\left(R_{\oplus}\right)$ & 6.11 & \pm 0.20 & $\mathrm{~b}, \mathrm{e}$ \\
\hline Planet mass, $M_{p}\left(M_{\oplus}\right)$ & $<120(3 \sigma)$ & & $\mathrm{b}, \mathrm{g}$ \\
\hline Planet mean density, $\rho_{p}\left(\mathrm{~g} \mathrm{~cm}^{-3}\right)$ & $<3.0(3 \sigma)$ & & $\mathrm{b}, \mathrm{e}, \mathrm{g}$ \\
\hline Sky-projected stellar obliquity, $\lambda(\mathrm{deg})$ & -110 & $-14,+22$ & $\mathrm{~h}$ \\
\hline Sky-projected stellar rotation velocity, $v \sin i_{\star}\left(\mathrm{km} \mathrm{s}^{-1}\right)$ & 5.6 & \pm 0.8 & $\mathrm{~h}$ \\
\hline Inclination of stellar rotation axis (deg) & 138 & \pm 7 & $\mathrm{i}$ \\
\hline Stellar obliquity, $\psi(\mathrm{deg})$ & 145 & $-14,+9$ & $\mathrm{j}$ \\
\hline
\end{tabular}

Notes. Each quoted result represents the median of the a posteriori probability distribution derived from the MCMC, marginalized over all other parameters. The confidence limits enclose $68.3 \%$ of the probability, and are based on the $15.85 \%$ and $84.15 \%$ levels of the cumulative probability distribution.

${ }^{a}$ Based on the SPC analysis of the spectra and the $Y^{2}$ models, using the gyrochronology age as a constraint (see Section 3).

$\mathrm{b}$ Based on the SPC analysis of the spectra and the $\mathrm{Y}^{2}$ models (see Section 3). The stellar density is given in units of $\rho_{\odot}=1.408 \mathrm{~g} \mathrm{~cm}^{-3}$.

${ }^{\mathrm{c}}$ Based on the periodogram of the Kepler photometric time series (see Section 3.1).

${ }^{\mathrm{d}}$ Based on the Keck/HIRES spectrum (see Section 3.1).

${ }^{\mathrm{e}}$ Based on the analysis of the transit light curves (see Section 4.2).

${ }^{\mathrm{f}}$ Based on the combination of transit parameters, orbital period, and mean stellar density (see Section 4.3).

g Based on the analysis of the Keck radial velocities, assuming zero eccentricity (see Section 4.4).

${ }^{\mathrm{h}}$ Based on the analysis on the RM effect (see Section 5).

i Based on the combination of $P_{\text {rot }}, R_{\star}$, and $v \sin i_{\star}$ (see Section 6.1).

${ }^{j}$ Based on the analysis of the RM effect and starspot-crossing events (see Section 6.2).

\subsection{Transit Analysis}

To obtain accurate transit parameters, we needed to correct for the effects induced by starspots (see, e.g., Czesla et al. 2009; Carter et al. 2011; Sanchis-Ojeda et al. 2012). We chose to work only with the short-cadence data, since the 30 minute time sampling of long-cadence data is too coarse to allow a clear identification of the spot-crossing events. We defined the transit window as an interval of four hours centered on the expected transit time. The out-of-transit (OOT) portion was defined as the two hours preceding the transit window plus the two hours following the transit window, giving a total of four hours of data. First, the data from each transit window were normalized such that the OOT data had a median flux of unity. Figure 2 shows an illustrative example. We visually inspected the 96 transit light curves and identified 145 spot-crossing events. All of them were temporary brightenings (rather than fadings), implying that the detectable spots on the surface of Kepler-63 have a temperature lower than the photosphere. This is in accordance with a general trend observed for very active stars (Foukal 1998). In order to properly estimate the transit parameters, the data points within these anomalies were assigned zero weight in the fits. More 


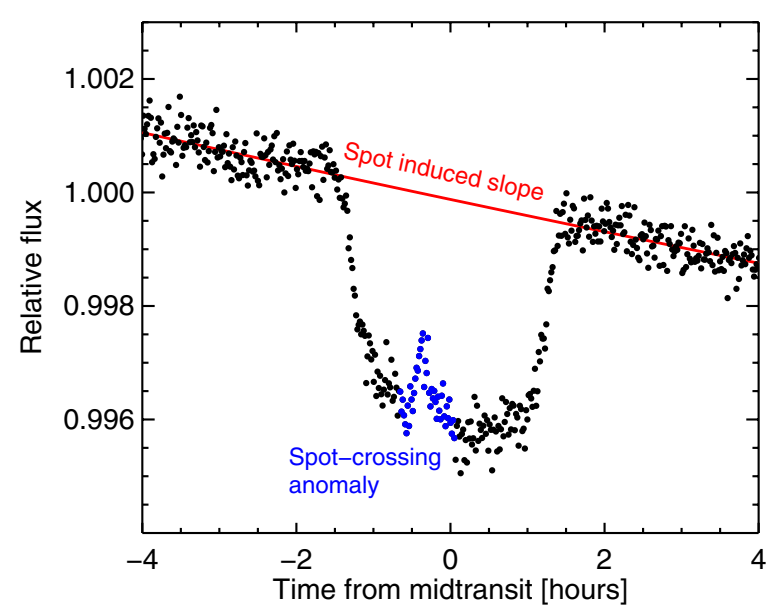

Figure 2. Example of the effects of starspots on transit signals. The black dots are Kepler data points for a particular transit. Spot-crossing anomalies (blue dots) are identified visually and masked out. Variability on the longer timescale is modeled as a second-order polynomial (red line).

(A color version of this figure is available in the online journal.)

than $25 \%$ of the in-transit data points were assigned zero weight, which speaks to the high level of activity on the particular region that the planet is transiting.

Figure 2 also shows the effect of the longer-term variations in stellar flux. Large starspots combined with the short rotation period introduce strong gradients in the OOT flux, and occasionally significant curvature, especially if the transit happens near a flux minimum or maximum. As long as we have removed the spot-anomalies correctly, the observed flux can be described as (cf. Carter et al. 2011)

$$
F(t)=F_{0}[1-\epsilon(t)]-\Delta F(t),
$$

where $F_{0}$ is the stellar flux in the absence of transits or spots, $\epsilon(t)$ is the fractional loss of light due to starspots, and $\Delta F(t)$ is the flux blocked by the planet. The function $\epsilon(t)$ is responsible for the overall gradient in time throughout the transit (see the red line in Figure 2). To obtain the normalized flux, we divide Equation (1) by $F_{0}[1-\epsilon(t)]$. We then model the OOT variation by a second-order polynomial in time, giving

$$
f(t) \approx 1+c_{0}+c_{1}\left(t-t_{c}\right)+c_{2}\left(t-t_{c}\right)^{2}-\frac{1}{1-\epsilon\left(t_{c}\right)} \frac{\Delta F(t)}{F_{0}},
$$

where the OOT variation is described by a second-order polynomial in time, and $\epsilon\left(t_{c}\right)$ represents the relative flux lost due to spots at the time of transit $t_{c}$. To determine the coefficients $c_{i}$, we fitted a second-order polynomial to the OOT portion of the data. We then subtracted the best-fitting polynomial from the data in the entire transit window, to "rectify" the data. The loss of light due to the planet, $\Delta F(t) / F_{0}$, was assumed to be the same for all transits, but each transit was assigned an independent value of $\epsilon\left(t_{c}\right)$. To avoid having to fit all the data with hundreds of parameters, we performed the transit modeling in four stages, described below.

\subsubsection{Step 1. Initial Folded Light Curve Analysis}

First, we needed good initial guesses for the transit parameters. We created a phase-folded light curve based on the normalized transits, using the orbital period from Batalha et al. (2013). We averaged the phase-folded light curve into four-minute bins, chosen to improve computation speed without a significant loss

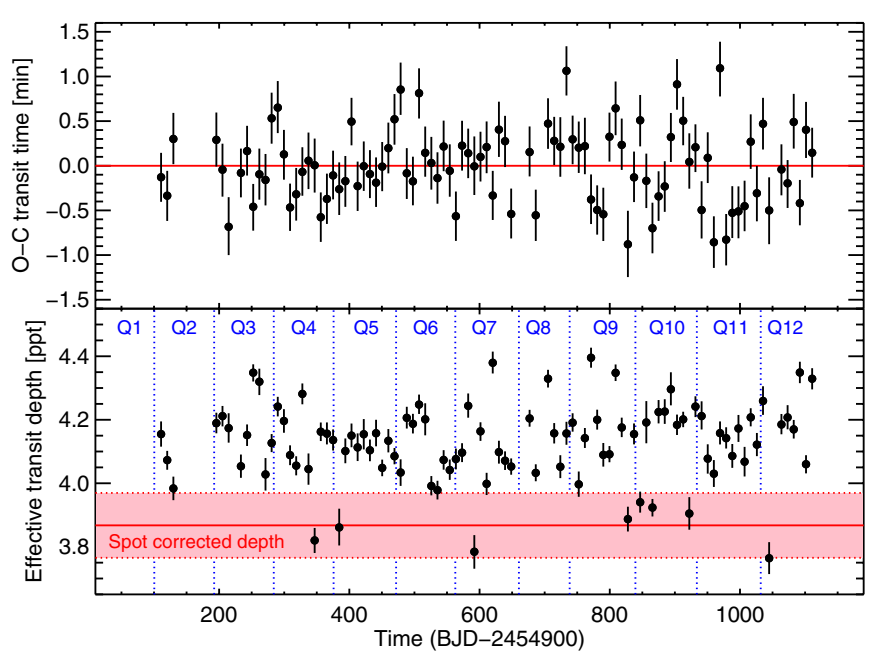

Figure 3. Variations in transit times and apparent depths. Top: residuals of a linear fit to the transit times of Kepler-63b. The excess of scatter is significant $\left(\chi^{2}=202\right.$ with 94 degrees of freedom) and is likely due to uncorrected spot-crossing events. Bottom: the black dots represent the effective depth of individual transits in parts per thousand (ppt). No strong correlation with the quarter is observed, suggesting that the variability is mostly due to starspots as opposed to variable amounts of blended flux. The final adopted value for the transit depth (red line) and its $1 \sigma$ confidence interval (shaded region) were obtained by assuming that the star is nearly spot-free during the transits with the smallest effective depth (see Section 4.2).

(A color version of this figure is available in the online journal.)

of accuracy. At this stage, we ignored the transit-to-transit variations and simply modeled the folded light curve with an idealized Mandel \& Agol (2002) model, the free parameters being $\left(R_{p} / R_{s}\right)^{2}, R_{s} / a$, the impact parameter $b$, and two linear combinations of the quadratic limb-darkening coefficients (chosen to minimize correlations as recommended by Pál 2008).

\subsubsection{Step 2. Individual Transit Analysis}

Next, we wanted to obtain individual transit times and depths. The five parameters of the best-fitting model from step 1 were held fixed, and the data from each transit window were fitted with three additional parameters: the time of transit $t_{c}$, the linear coefficient $c_{1}$ from Equation (2), and the spot-coverage factor $\epsilon\left(t_{c}\right)$ (which for brevity we hereafter denote simply $\epsilon$ ). The linear coefficient $c_{1}$ was allowed to vary because it is covariant with the transit time. We assigned an uncertainty of $279 \mathrm{ppm}$ to each individual SC data point, as this is the standard deviation of the OOT portion of the unbinned folded light curve. We found the best-fitting model for each individual transit light curve and used a Markov Chain Monte Carlo (MCMC) algorithm to explore the allowed parameter space.

We fitted a linear function of epoch to the transit times and used it to estimate the orbital period and a particular transit time (chosen to be the first transit observed with short cadence). Figure 3 shows the residuals between the observed and calculated transit times. There is no clear structure, but the fit has $\chi^{2}=202$ with 94 degrees of freedom, suggesting that the uncertainties on the transit times have been underestimated. We attribute this excess scatter to uncorrected effects of stellar spots (see, e.g., Sanchis-Ojeda et al. 2011; Oshagh et al. 2013b), although transit-timing variations could also be present due to another planet orbiting the same star (Agol et al. 2005; Holman \& Murray 2005; Nesvorný et al. 2012). To account for the excess scatter, we enlarged the uncertainties in the orbital period and the transit epoch by $46 \%$ (such that $\chi^{2}=N_{\text {dof }}$ ). 
In our procedure, each transit is associated with a particular value of $\epsilon$, but it is a more common practice to report an effective depth for each transit. The transit depth obtained in this way would be equivalent to the transit depth fixed in step 1 , shared by all transits, divided by $1-\epsilon$. In the lower panel of Figure 3 , we plot this effective depth for each transit. The variability of the apparent transit depths is as high as $10 \%$. There is no clear correlation of the apparent depths with the Kepler quarter, implying that contamination from background stars, if present, must be very small or common to all the photometric apertures used in the different quarters. The observed variability is most likely induced by starspots.

\subsubsection{Step 3. Choice of the Baseline Transit Depth}

In order to obtain the final transit parameters, we readjusted the scale by which $\epsilon$ is measured, thereby renormalizing the transits. The distribution of spot coverage factors $(\epsilon)$ had a mean near zero and standard deviation close to $3 \%$. This choice would make sense if the effects of dark starspots and bright plages were comparable on average. However, we did not detect any plage-crossing events, whereas we did detect many spotcrossing events. Assuming that dark starspots dominate the stellar flux variations, the true loss of light should always be positive, and the shallowest effective transit depth should occur when the star has the smallest spot coverage. For that particular transit the true $\epsilon \approx 0$ and the rest of spot coverage factors are positive (Carter et al. 2011; Sanchis-Ojeda et al. 2012).

A simple approach would be to identify the transit with the smallest value of $\epsilon$, and subtract that value from the entire distribution of $\epsilon$ values. We chose instead a more robust method that does not depend entirely on a single $\epsilon$ value. First, we removed outliers from the distribution of $\epsilon$ factors using a $3 \sigma$ clipping algorithm. Then, we assumed that the distribution is Gaussian and computed the standard deviation of the remaining $\epsilon$ factors. Finally, we shifted the distribution of $\epsilon$ values to force the median of the distribution to be two standard deviations above zero. This procedure ensures that most of the $\epsilon$ factors are positive.

\subsubsection{Step 4. Final Transit Parameters}

To obtain the final transit parameters, we used the $\epsilon$ values determined in step 3 to renormalize each transit light curve. The intention was to correct all of the transit signals to have a uniform shape, similar to what one would observe if the planet were transiting a spot-free star. The transit data were then folded using the newly calculated linear ephemeris, binned to have a time sampling of one minute, and modeled with the same five-parameter transit model used in step 1. Table 2 gives the final parameter values, with uncertainties estimated using an MCMC algorithm. The uncertainty in the transit depth was enlarged beyond the statistical uncertainty, to take into account the procedure we have described in step 3 . We used the width of the distribution of measured depths as the measure of systematic uncertainty in the transit depth. The final value of the depth with the enlarged uncertainty is depicted in Figure 3.

\subsection{Orbital Eccentricity}

The orbital period of Kepler-63b is long enough that it is not safe to assume the orbital eccentricity has been damped by tides to a negligible level. We attempted to learn the orbital eccentricity in two different ways: by searching for occultations (secondary eclipses); and by using the mathematical relationship between the transit parameters, the mean stellar density, and the orbital eccentricity and argument of pericenter.

If the orbit were circular, occultations would occur halfway in between transits, with a duration equal to that of the transits. For an eccentric orbit, the timing and duration of the secondary eclipse depend on the eccentricity $e$ and the argument of periastron $\omega$ (see, e.g., Winn 2011). A grid search was performed to detect the occultation, but the result was negative. This is not surprising, since the occultation depth would be of the order of $\left(R_{p} / a\right)^{2}=10 \mathrm{ppm}$, which is below our level of detectability.

One can also obtain information about the eccentricity of the orbit by combining the orbital period, scaled semimajor axis, and mean stellar density $\rho_{\star}$ (see, e.g., Dawson \& Johnson 2012). We compute

$$
\frac{1+e \sin \omega}{\sqrt{1-e^{2}}} \approx \frac{2 \sqrt{R_{p} / R_{\star}}}{\sqrt{T_{14}^{2}-T_{23}^{2}}}\left(\frac{3 P}{G \pi^{2} \rho_{\star}}\right)^{1 / 3}=0.92 \pm 0.02,
$$

where $T_{14}$ is the duration between first and fourth contact, and $T_{23}$ is the duration between second and third contact. This expression is close to unity when the eccentricity is low. Based on the measured values of $T_{14}, T_{23}, P$, and $R_{p} / R_{\star}$, and the estimated value of $\rho_{\star}$ from Section 3.2, we find $e<0.45(3 \sigma)$. The formal $68.3 \%$ confidence interval is $0.08-0.27$. Thus the eccentricity is not likely to be very high, but moderate values cannot be excluded.

\subsection{Radial Velocity Analysis}

For the RV analysis, we used the seven Keck RVs obtained before the transit night. We also took the mean of all the pretransit RVs from the night of 2011 August 20/21, and treated this mean velocity as a single additional data point. A planet somewhat smaller than Jupiter in a 10 day orbit should induce an RV signal with a semiamplitude of the order of tens of $\mathrm{m} \mathrm{s}^{-1}$. For a chromospherically quiet star, eight RVs with a precision of a few $\mathrm{m} \mathrm{s}^{-1}$ would have been enough to determine the mass of the planet. However, in the case of Kepler-63, we expect spurious RVs of the order of tens of $\mathrm{m} \mathrm{s}^{-1}$, the product of $v \sin i_{\star}$ and the fractional photometric variability. This greatly complicates the mass determination.

The top panel of Figure 4 shows that the stellar flux variations during the RV observations were approximately sinusoidal. The simplest explanation is that a single large dark spot was always on the visible side of the star. In such configurations, it is possible to estimate the spurious radial velocity $V_{\text {spot }}$ due to starspots using the so-called $F F^{\prime}$ method of Aigrain et al. (2012). Those authors showed that the spurious RV can be approximated by a function of the normalized stellar flux $f(t)$ and its derivative,

$$
V_{\text {spot }}\left(\epsilon, \kappa \delta V_{c}\right)=\dot{f}(t)[1-f(t)] \frac{R_{\star}}{\epsilon}+[1-f(t)]^{2} \frac{\kappa \delta V_{c}}{\epsilon},
$$

where the normalization is such that the $f(t)=1$ level is one standard deviation above the maximum observed flux. There are two free parameters: $\epsilon$ is the relative loss of light due to the spot if it were situated at the center of the stellar disk, and $\kappa \delta V_{c}$ specifies the alteration of the convective blueshift due to the spot. With this ingredient, our model for the RV signal was

$$
V_{\text {calc }}=-K \sin \left[n\left(t-t_{c}\right)\right]-V_{\text {spot }}\left(\epsilon, \kappa \delta V_{c}\right)+\gamma .
$$

In this formula, we have assumed a circular orbit for the planet, with $K$ being the planet-induced RV semiamplitude and $t_{c}$ the 


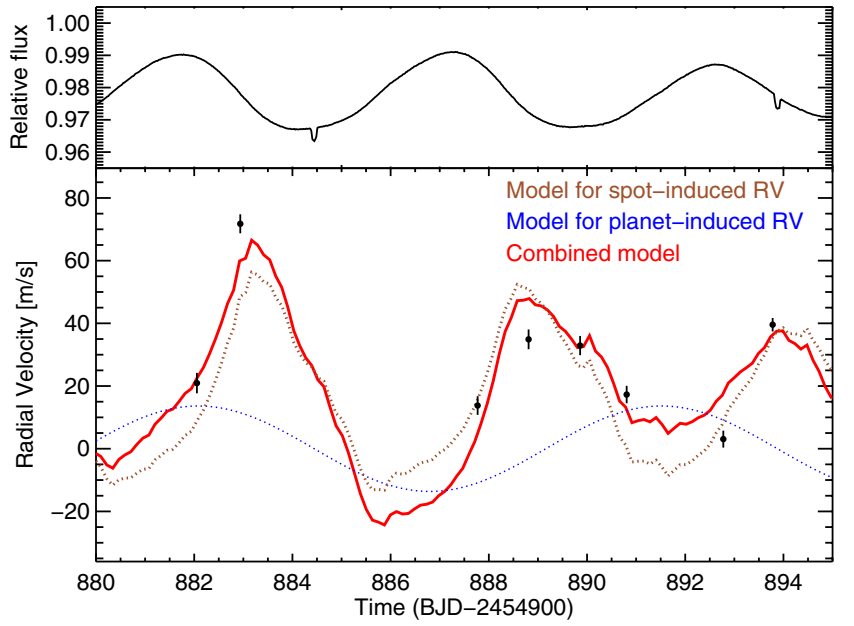

Figure 4. Radial-velocity analysis. Top: relative stellar flux of Kepler-63 during the radial-velocity observations. Bottom: black dots represent the radial-velocity observations, with vertical bars indicating the internally estimated measurement uncertainties (with no "jitter" term added). The red line represents the optimized model, which is the sum of a sinusoidal function representing the planetary signal (blue line) and the $F F^{\prime}$ model representing the spurious radial velocity due to rotating starspots (brown line).

(A color version of this figure is available in the online journal.)

time of transit. The parameter $\gamma$ represents a constant offset. The mean motion $n$ is defined as $2 \pi / P_{\text {orb }}$. Both $P_{\text {orb }}$ and $t_{c}$ are known precisely from the transit analysis.

We optimized the model parameters through a standard leastsquares fit. Figure 4 displays the data and the optimized model. Given the typical measurement uncertainty of $2.5 \mathrm{~m} \mathrm{~s}^{-1}$, the minimum $\chi^{2}$ was 78.6 with 4 degrees of freedom. This poor fit is at least partly due to the simplicity of the spot model (which assumes that there is only one small spot on the surface). By adding a "jitter" term of $12.5 \mathrm{~m} \mathrm{~s}^{-1}$ in quadrature to the measurement uncertainties, we obtain $\chi^{2}=N_{\text {dof }}$. We use this jitter term and an MCMC algorithm to determine credible intervals for the model parameters, from which we derive a planet mass of $M_{p}=45 \pm 26 M_{\oplus}$. The $3 \sigma$ upper bound is $120 M_{\oplus}$.

Given the severe limitations of this analysis-the weak detection, the imperfect fit of the $F F^{\prime}$ model, and the assumption of zero eccentricity - we do not claim to have detected the RV signal due to the planet. Rather, we interpret the results as a coarse upper bound on the mass of the transiting object, placing it within the planetary regime.

\section{SKY-PROJECTED OBLIQUITY FROM THE RM EFFECT}

The RM effect is more easily detected than the orbital motion, mainly because the timescale of the RM effect is much shorter than the rotation period of the star, allowing a clean separation between the RM effect and the spurious starspot-induced RVs (see, e.g., Gaudi \& Winn 2007). Prior to the Keck observations, we had performed enough spot modeling to be able to predict that the anomalous Doppler shift would be a pure redshift throughout the transit. The results of the RM observations, displayed in Figure 5, confirmed this prediction. However, the Kepler photometry of the same transit (shown in the bottom panel of Figure 5) revealed at least three spot-crossing events, complicating the modeling.

To model the RM effect, one needs the usual parameters describing the loss of light as well as four additional parameters: $v \sin i_{\star}$ and $\lambda$ to describe the amplitude and shape of the signal,

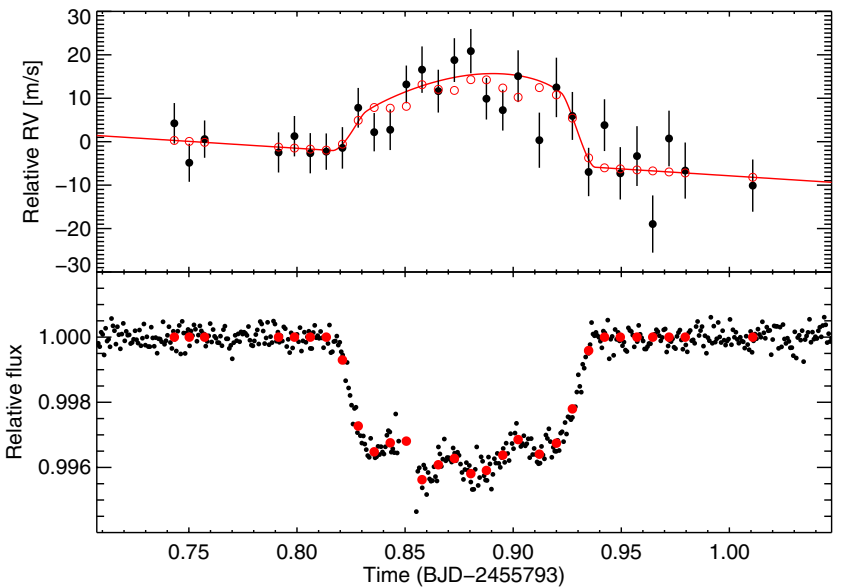

Figure 5. Evidence for a high obliquity based on the RM effect. Top: solid dots are the measured radial velocities. The signal is a redshift throughout the transit, applying a high obliquity, as opposed to the red-then-blue signal of a low-obliquity system. The open dots represent the best-fitting model. The red curve shows a model with the same geometric parameters but with the loss of light appropriate for a spotless star, to illustrate what one might have observed in the absence of starspots. Bottom: transit observations in SC mode obtained with Kepler. The black dots represent the data and the red dots represent the binned light curve used to model the RM effect.

(A color version of this figure is available in the online journal.)

and a slope $\dot{\gamma}$ and offset $\gamma$ to account for the orbital motion of the star. Usually the loss of light is computed based on the transit parameters $b, R_{\mathrm{s}} / a, R_{\mathrm{p}} / R_{\star}$ and the time of transit. Here, given the presence of spot-crossing anomalies, we chose to take the loss of light directly from the Kepler photometric time series, since this naturally takes the anomalies into account, and the cadence and precision are more than sufficient for our purpose. To obtain the loss of light corresponding to each point in the RV time series, we averaged the corresponding Kepler photometric data points. The final averaged light curve is shown in the lower panel of Figure 5 as the sequence of red dots.

The anomalous RV was then computed with the formulas of Hirano et al. (2011), using the planet position and loss of light as inputs. This code takes into account the effects of macroturbulence, pressure broadening, and instrumental broadening. Model fitting and parameter estimation were performed using the protocols of Albrecht et al. (2012). In particular, we imposed Gaussian priors on $T_{14}$ and $T_{12}$, based on the parameters reported in Table 2 . We also used the parameter combinations $\sqrt{v \sin i_{\star}} \sin \lambda$ and $\sqrt{v \sin i_{\star}} \cos \lambda$ rather than $v \sin i_{\star}$ and $\lambda$, to minimize correlations. The uncertainty in each RV data point was taken to be the quadrature sum of the internally estimated uncertainty and $4.8 \mathrm{~m} \mathrm{~s}^{-1}$, the value for which $\chi^{2}=N_{\text {dof }}$. The result for the sky-projected obliquity is $\lambda=-110_{-14}^{+22} \mathrm{deg}$.

There are some other interesting results of this analysis. We find the projected rotation speed to be $v \sin i_{\star}=5.6 \pm$ $0.8 \mathrm{~km} \mathrm{~s}^{-1}$, in agreement with the value obtained from the basic spectroscopic analysis (see Section 3.2). The result for the OOT velocity slope $\dot{\gamma}=-30 \pm 15 \mathrm{~m} \mathrm{~s}^{-1}$ day $^{-1}$ can be translated into an estimate of the velocity semiamplitude due to the planet, using the orbital period and assuming a circular orbit. The result is $K_{\mathrm{RM}}=-\dot{\gamma} P_{\text {orb }} / 2 \pi=40 \pm 20 \mathrm{~m} \mathrm{~s}^{-1}$. This slope is compatible within the uncertainties with the measured $K$ from the RV analysis (approximately $15 \mathrm{~m} \mathrm{~s}^{-1}$ ), although the uncertainties are large, and the effects of spots were not taken into account in this determination of $K_{\mathrm{RM}}$. In this case, by chance, the transit happened about a quarter of a rotation 

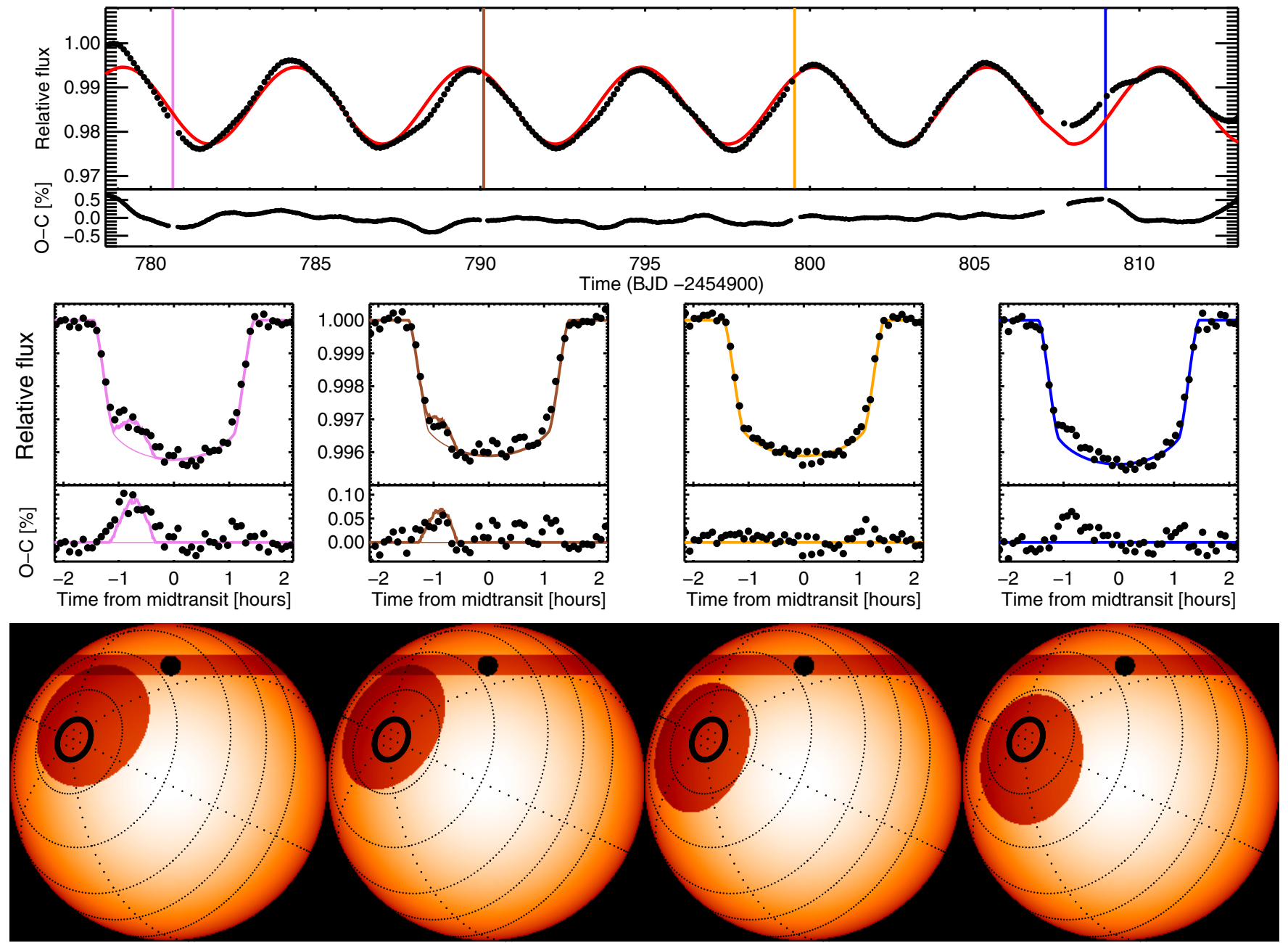

Figure 6. Evidence for a large obliquity from a single-spot model. Top: relative flux of Kepler-63 (black dots) over a time range spanning four transits. The transit times are marked with vertical lines. The red line represents the best-fitting model with a single starspot. Center: transit light curves, with five minute sampling. The thick curves represent the best-fitting model with a single starspot; the thin curves show the model with the spot darkening set equal to zero. The model accounts for the two largest spot-crossing anomalies but the residuals indicate that more spots are present. Bottom: locations of the spot, transit chord, and planet at midtransit, according to the best-fitting model.

(A color version of this figure is available in the online journal.)

cycle before a flux minimum, which is when the spot-induced spurious acceleration is expected to be small.

\section{OBLIQUITY MEASUREMENT FROM STARSPOTS}

\subsection{Stellar Inclination from $v \sin i_{\star}$}

We combined the values of the rotation period $P_{\text {rot }}$, stellar radius $R_{\star}$, and sky-projected stellar rotation velocity $v \sin i_{\star}$ to obtain $\sin i$, the inclination of the stellar rotation axis with respect to the line of sight. Based on the values given in Table 2, the stellar rotation velocity is $v=2 \pi R_{\star} / P_{\text {rot }}=$ $8.4 \pm 0.2 \mathrm{~km} \mathrm{~s}^{-1}$. This is significantly larger than $v \sin i_{\star}=$ $5.6 \pm 0.8 \mathrm{~km} \mathrm{~s}^{-1}$ obtained from the analysis of the RM effect, implying $\sin i_{\star}<1$. The implied stellar inclination angle is either $42 \pm 7 \mathrm{deg}$ or $138 \pm 7 \mathrm{deg}$. As we will see in the next section, the latter value of the stellar inclination is favored. Since Kepler-63b is transiting with an orbital inclination of $87.81 \pm 0.02 \mathrm{deg}$, this simple analysis demonstrates that the star has a high obliquity, independently of the RM effect.

We used the marginalized posterior for $\lambda$ obtained in the last section, as well as those for the stellar and orbital inclinations
(Table 2), to obtain the true obliquity $\psi$. Using the formula from Fabrycky \& Winn (2009), the result is $\psi=104_{-14}^{+9} \mathrm{deg}$.

\subsection{Sky-projected Obliquity from Spot-crossing Anomalies}

In principle, the obliquity of the system is also encoded in the pattern of photometric variability and spot-crossing anomalies, but in this case the anomalies are so numerous that the pattern has proven difficult to interpret unambiguously. Rather than attempt a rigorous independent determination of the obliquity, we discuss here a starspot model that at least demonstrates the compatibility between the photometric variability, the starspot-crossing events, and the preceding results for $\lambda$ and $i_{\star}$.

We focused our attention on a time interval when the overall photometric variability seemed relatively simple: a nearly sinusoidal pattern with peak-to-peak amplitude of about $1.5 \%$. This interval spans four consecutive transits, specifically epochs 71-74 (see Figure 6). A large and long-duration spot-crossing anomaly is seen in the first half of the first transit. We proceeded by assuming that this is the same spot that is producing the quasi-periodic stellar flux variation, and attempted to model all of the data under this premise. 


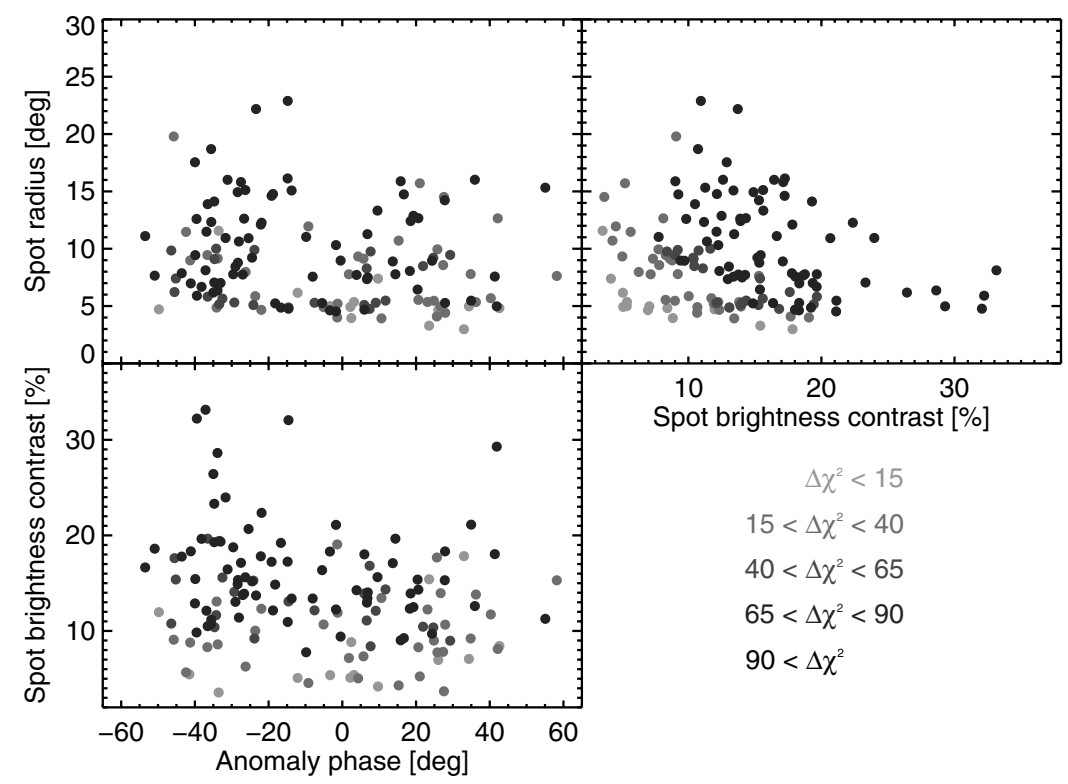

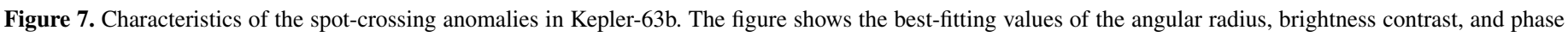
of the 145 spot-crossing anomalies that were identified. The darker dots represent more significant detections.

The orientation of the star was parameterized by the skyprojected obliquity $\lambda$ and the inclination angle $i_{\star}$. The rotation period was a free parameter, which was tightly constrained by the quasi-periodic variability. A Gaussian prior constraint was imposed on $v \sin i_{\star}$ based on the results of Section 6.1.

We modeled the OOT variability using the Dorren (1987) equations for the loss of light due to a starspot. We fixed the limb-darkening coefficient to a value of 0.56 , which provides the best fit to the light curve constructed in Section 4.2. The spot's brightness contrast relative to the photosphere was taken to be a constant over the interval of the observations. The observed phase of the OOT variability specifies the spot longitude, and the stellar rotation period is also well constrained. Therefore, given particular choices for the orientation of the star and the spot latitude, we could calculate the location of the spot at any time, including the times of the four transits.

The transits were modeled using the geometric transit parameters from Section 4.2, and a pixelated stellar disk. At any particular time, we computed the sum of the intensities of all the pixels, some of which were darkened by the spot or hidden by the planet (Sanchis-Ojeda et al. 2011).

The best-fitting value for $\lambda$ was $-115^{\circ}$, in agreement with the result based on the RM effect. The model also prefers $i_{\star}=135^{\circ}$, selecting one of the two values for the inclination that were allowed by the analysis in Section 6.1. This constraint arises from the requirement that the spot must cross the transit chord before its closest approach to the center of the stellar disk. In the optimized model, the spot is large and resides near one of the rotation poles.

The simple one-spot model is therefore compatible with the overall photometric variability and the largest spot anomaly. The smaller spot anomaly during the second transit can also be attributed to this spot, as illustrated in Figure 6. Certainly, though, this model does not capture all of the sources of photometric variability: there are at least six other smaller anomalies that are not well-fitted, and which it does not seem worthwhile to try and model. The large anomaly in the fourth transit agrees in phase with the two other explained anomalies. It is possible that the same spot is responsible for this anomaly, if the spot has an irregular shape.

\section{STARSPOT CHARACTERISTICS AND MAGNETIC CYCLES}

As discussed in Section 4.2, we visually identified 145 spotcrossing anomalies. To study the position, sizes, and temperatures of the spots, we modeled the individual spot-crossing anomalies with the same pixelated spot model discussed in Section 6.2. The parameters describing each spot-crossing event were the spot's angular radius and brightness contrast, as well as the timing of the event, which we express as an "anomaly phase" ranging from $-90^{\circ}$ (ingress) to $90^{\circ}$ (egress). The other transit parameters, including the spot coverage factor $\epsilon$, were taken from the analysis of Section 4.2. To evaluate the significance of detection of the anomalies, we used $\Delta \chi^{2}$ between the best-fitting spot model and the best-fitting spot-free model.

There is a degeneracy between the modeled position and radius of a spot, because we lack the precision to measure the impact parameter between the planet and spot (and there is no reason to think the spot is perfectly circular). To avoid this degeneracy, we assumed that the planet passes through the center of the spot. It should be understood, then, that the "spot radius" in our model is really a measure of the length of the intersection between the spot and the transit chord.

Figure 7 shows the results. Unsurprisingly, the significance of detection increases with the size and the brightness contrast of the spots. The anomalies that appear in the first half of the transit (negative anomaly phase) are generally more significant and more abundant. The typical spot radius is $10^{\circ}$, and the largest spots have a radius of $15^{\circ}-20^{\circ}$. The typical brightness contrast is $15 \%-20 \%$. To produce a $20 \%$ brightness contrast in the Kepler bandpass (450-850 nm) would require an effective temperature approximately $300 \mathrm{~K}$ lower than the photosphere, assuming blackbody spectra.

Figure 8 shows the time evolution of the spot-crossing events. To create this figure, we subtracted a model for the loss of light due only to the planet, and then divided the residuals by the transit depth. The normalized residuals were then plotted as a function of both time (horizontal axis) and phase within the transit (vertical axis). Given the known orientation of the star, we can also translate the phase within the transit into a 


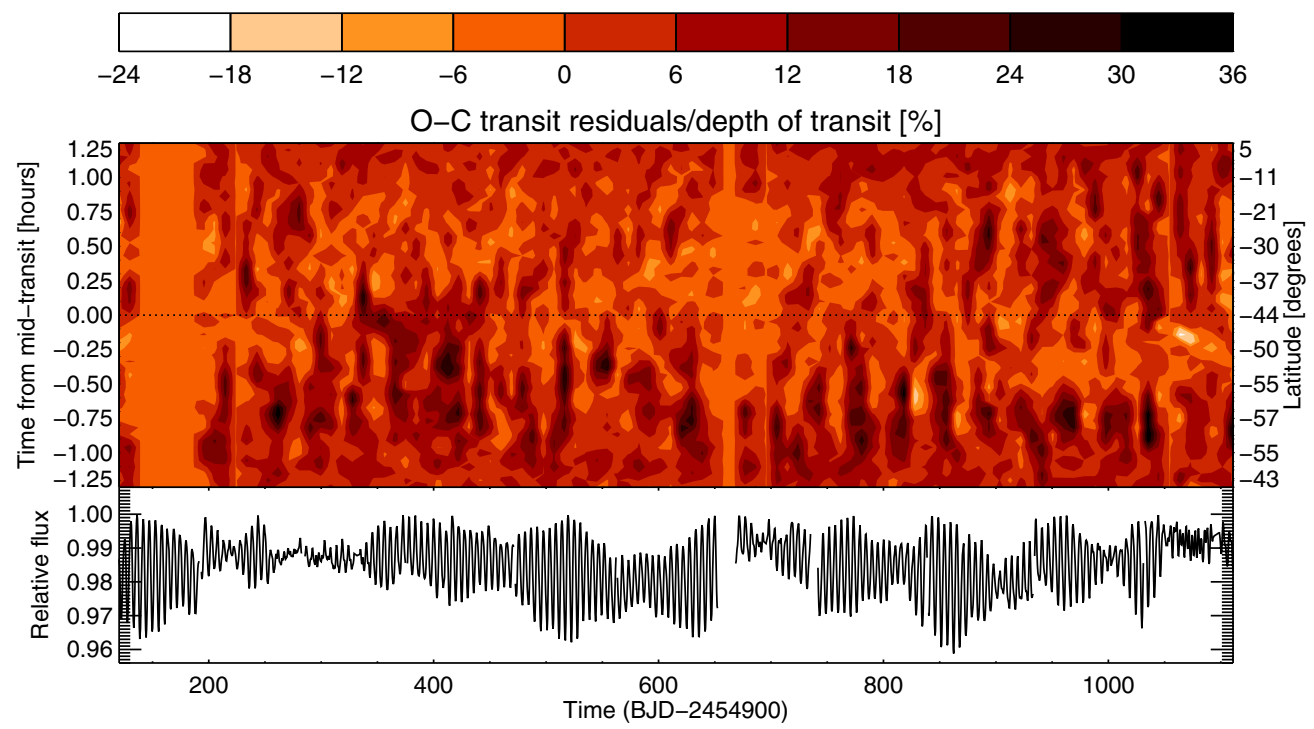

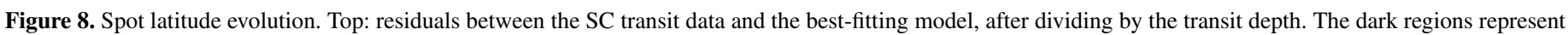

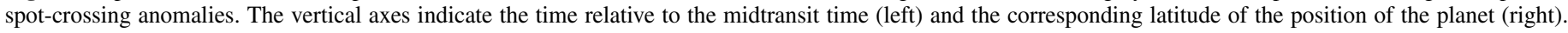

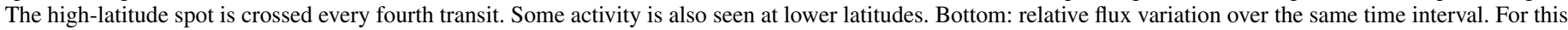
plot the data from each Kepler quarter were normalized by the maximum quarterly flux.

(A color version of this figure is available in the online journal.)

stellar latitude (second vertical axis). The transit chord spans latitudes from $-60^{\circ}$ to $5^{\circ}$, that is, a large portion of the southern hemisphere. We also note that the relation between the transit phase and the stellar latitude is nonlinear; indeed, some latitudes cross the transit chord in more than one location.

Spot-crossing events are visible as dark regions in this plot. Most of the activity is seen in the early portions of the transits. This indicates a long-lived polar active region. After the first few years of observations (starting at around day 875), spotcrossing anomalies began to appear in the second half of the transit, corresponding to lower stellar latitudes. Anomalies at mid-transit were comparatively rare, especially once the second half of the transit chord became active. Perhaps this is a sign that active regions tend to be segregated in latitude, with some activity at high polar latitudes and some at more equatorial latitudes.

These initial explorations of the spatial distribution of activity on Kepler-63 could be continued in the future by developing a multi-spot model, fitted to both the stellar flux variations and the spot-crossing anomalies (see, e.g., Bonomo \& Lanza 2012; Oshagh et al. 2013a). Here we have focused mainly on the anomalies, which provide snapshots of the transit chord every 9.4 days; there are undoubtedly some spots that are missed with this approach (Llama et al. 2012).

\section{DISCUSSION}

In this paper, we have presented Kepler-63b, a giant planet transiting a star on an orbit that is highly inclined with respect to the stellar equator. On the one hand, the star's high levels of chromospheric activity interfered with our ability to characterize the system through transit light curve analysis and RV monitoring. On the other hand, the Kepler data allowed us to partly correct for the effects of activity, and also to take advantage of the activity to determine the stellar rotation period, explore the spatial distribution of starspots, and perform a consistency check on the stellar obliquity that was determined via the RM effect.
The measurement of the planet's mass through RV measurements was unsuccessful because the spurious RVs caused by starspots were larger than the planet-induced signal. To measure the mass, a large body of additional RVs will be required, in a campaign that is carefully designed to try and separate the effects of rotating starspots and orbital motion. The information about the general spot characteristics presented in this paper may help in designing such a campaign.

The star's high obliquity corroborates the scenario proposed by Winn et al. (2010a) and Albrecht et al. (2012) in which hot Jupiters have orbital inclinations that are initially nearly random with respect to the stellar equator, and are eventually damped to low inclinations if the tidal interactions between the star and planet are sufficiently strong. In this scenario, a high obliquity is expected for Kepler-63, because even though the star is relatively cool and has a thick convective envelope (a factor leading to relatively rapid tidal dissipation), the orbital distance is relatively large. To be quantitative, we used the metric developed by Albrecht et al. (2012), in which binary-star data are used to calibrate tidal dissipation timescales. Figure 9 shows that the expected timescale for tidal dissipation for this system is in the regime where random alignment is observed among the other close-in giant planets. The fact that the star is young also helps us to understand why it has not yet been realigned (Triaud 2011). This measurement is interesting because among planet-hosting stars with measured obliquities, only HAT-P-11b is comparable to Kepler-63b in size and orbital period, being smaller $\left(4.7 R_{\oplus}\right)$ than Kepler-63b but also having a shorter orbital period (4.9 days).

A proposed interpretation for these findings is that hot Jupiters begin far away from the star, beyond the snow line, where it is easier to understand their formation. The initial obliquity of the system is low, as a consequence of the formation of the entire system from a single disk of gas and dust. Then, dynamical interactions, such as planet-planet scattering (Rasio \& Ford 1996) or Kozai cycles induced by the influence of a distant companion (Fabrycky \& Tremaine 2007), move the planet into a highly eccentric orbit with a more random orientation. 


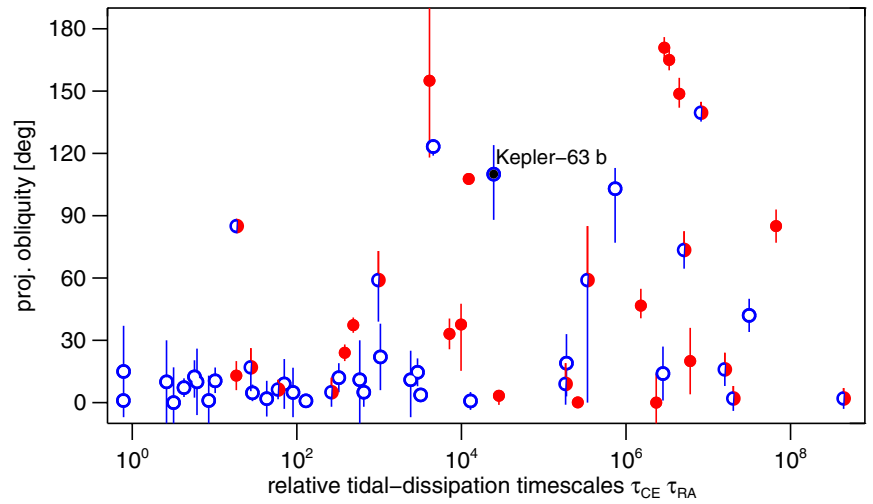

Figure 9. Sky-projected obliquities as a function of relative timescale for tidal dissipation. See Albrecht et al. (2012) for the original figure on which this is based, and for details on its construction. In short, the relative tidal dissipation timescale is assumed to be proportional to $q^{2}\left(a / R_{\star}\right)^{6}$ for stars with $T_{\text {eff }}<6250 \mathrm{~K}$ (blue dots) and proportional to $q^{2}(1+q)^{5 / 6}\left(a / R_{\star}\right)^{8.5}$ for hotter stars (red dots), where $q$ is the planet-to-star mass ratio. The hot and cool stars are placed on the same scale using an empirical calibration based on observations of binary-star circularization periods. The dots with both colors represent stars for which the measured $T_{\text {eff }}$ straddles the boundary. Lower obliquities are seen in systems with relatively rapid tidal dissipation, suggesting that tides are responsible for damping stellar obliquities. Even though Kepler-63 is a cool star which is relatively dissipative, the orbital distance is large enough that tides are relatively weak, and the high stellar obliquity fits well with this observed trend. In addition to Kepler-63, this plot features new and updated values of $\lambda$ for CoRoT-11b (Gandolfi et al. 2012), WASP-19b (Tregloan-Reed et al. 2013), WASP-32b and WASP-38b (Brown et al. 2012), and HAT-P-17b (Fulton et al. 2013).

(A color version of this figure is available in the online journal.)

In this highly eccentric orbit, the planet passes very close to the star, where tidal interactions are significant. This tidal interactions will circularize and shrink the orbit and, if they are strong enough, will realign the spin axis of the star with the orbital angular momentum. In the context of this theory, pursuing obliquity measurements for systems with smaller planets and longer orbital periods is interesting because at a certain point those planets might have been able to form in situ, leading to an expectation of a population of well-aligned systems.

Rogers et al. (2012) have proposed that at least some of the high obliquities might have nothing to do with planet formation per se but are instead the consequence of reorientation of stellar photospheres due to the redistribution of angular momentum by internal gravity waves. Their theory is applicable to stars with radiative envelopes, and is therefore not applicable to Kepler-63, nor to the other three cool stars with high obliquities that are seen in Figure 9.

In addition to measuring the obliquity of Kepler-63, we have confirmed that the planet is passing in front of a large, dark, persistent spot (or group of spots) located near one of the star's rotation poles. Such spots are not seen on the present-day Sun, where the spot latitudes follow an $11 \mathrm{yr}$ cycle in which they start appearing at medium latitudes $\left(30^{\circ}-40^{\circ}\right)$ and end up appearing near the equator (for a review, see Solanki 2003). However, there was previous evidence that polar spots are common around young Solar analogs. This was based on simulations of magnetic activity (Brown et al. 2010; Schrijver \& Title 2001) as well as empirical evidence from Doppler imaging of young and rapidly rotating stars such as EK Dra (Strassmeier \& Rice 1998). Even though such polar spots were detected in different occasions and with different techniques (Strassmeier et al. 1991), and multiple tests were performed to validate the technique (Unruh
\& Collier Cameron 1995; Bruls et al. 1998), an independent confirmation using a different method was previously lacking. Our study provides further evidence for these types of spots, through a direct method based on periodic occultations of the spots by a planet with a well-understood geometry. The current information gathered about stellar spots on Kepler-63, and future studies that could analyze the information from stellar flux variations, may provide useful information about the activity of young Sun-like stars. It would also be interesting to find additional active stars with transiting planets in the Kepler database, as a probe not only of stellar obliquities but also starspot characteristics and evolution.

We thank the anonymous referee for numerous insightful suggestions that led to major improvements in this paper. We also thank Andrew Collier Cameron, Bryce Croll, and Benjamin Brown for helpful discussions, and the entire Kepler team for the success of the mission. R.S.O. and J.N.W. acknowledge NASA support through the Kepler Participating Scientist program. Kepler was competitively selected as the tenth Discovery mission. Funding for this mission was provided by NASA's Science Mission Directorate. The data presented in this article were obtained from the Mikulski Archive for Space Telescopes (MAST). STScI is operated by the Association of Universities for Research in Astronomy, Inc., under NASA contract NAS5-26555. Support for MAST for non-HST data is provided by the NASA Office of Space Science via grant NNX09AF08G and by other grants and contracts. J.A.C. acknowledges support by NASA through a Hubble Fellowship (grant HF-51267.01-A). R.I.D. is supported by the NSF-GRFP (DGE-1144152). J.A.J. is supported by generous grants from the Alfred P. Sloan Foundation and the David and Lucile Packard Foundation. T.L.C., W.J.C., and G.R.D. acknowledge the support of the UK Science and Technology Facilities Council (STFC). Funding for the Stellar Astrophysics Centre is provided by The Danish National Research Foundation (grant agreement DNRF106). This research was partly supported by the ASTERISK project (ASTERoseismic Investigations with SONG and Kepler) funded by the European Research Council (grant agreement No. 267864). G.T. acknowledges partial support for this work from NSF grant AST-1007992.

\section{REFERENCES}

Agol, E., Steffen, J., Sari, R., \& Clarkson, W. 2005, MNRAS, 359, 567

Aigrain, S., Pont, F., \& Zucker, S. 2012, MNRAS, 419, 3147

Albrecht, S., Winn, J. N., Johnson, J. A., et al. 2012, ApJ, 757, 18

Barnes, S. A. 2007, ApJ, 669, 1167

Basri, G., Walkowicz, L. M., Batalha, N., et al. 2011, AJ, 141, 20

Batalha, N. M., Rowe, J. F., Bryson, S. T., et al. 2013, ApJS, 204, 24

Batalha, N. M., Rowe, J. F., Gilliland, R. L., et al. 2010, ApJL, 713, L103

Bonomo, A. S., \& Lanza, A. F. 2012, A\&A, 547, A37

Borucki, W. J., Koch, D., Basri, G., et al. 2010, Sci, 327, 977

Brown, B. P., Browning, M. K., Brun, A. S., Miesch, M. S., \& Toomre, J. 2010, ApJ, 711, 424

Brown, T. M., Latham, D. W., Everett, M. E., \& Esquerdo, G. A. 2011, AJ, 142,112

Brown, D. J. A., Collier Cameron, A., Díaz, R. F., et al. 2012, ApJ, 760, 139 Bruls, J. H. M. J., Solanki, S. K., \& Schuessler, M. 1998, A\&A, 336, 231 Bryson, S. T., Jenkins, J. M., Gilliland, R. L., et al. 2013, arXiv:1303.0052 Buchhave, L. A., Latham, D. W., Johansen, A., et al. 2012, Natur, 486, 375 Butler, R. P., Marcy, G. W., Williams, E., et al. 1996, PASP, 108, 500 Carter, J. A., Winn, J. N., Holman, M. J., et al. 2011, ApJ, 730, 82

Chaplin, W. J., Sanchis-Ojeda, R., Campante, T. L., et al. 2013, ApJ, 766, 101 Czesla, S., Huber, K. F., Wolter, U., Schröter, S., \& Schmitt, J. H. M. M. 2009, A\&A, 505, 1277

Dawson, R. I., \& Johnson, J. A. 2012, ApJ, 756, 122 
Deming, D., Sada, P. V., Jackson, B., et al. 2011, ApJ, 740, 33

Désert, J.-M., Charbonneau, D., Demory, B.-O., et al. 2011, ApJS, 197, 14

Dittmann, J. A., Close, L. M., Green, E. M., \& Fenwick, M. 2009, ApJ, 701,756

Djupvik, A. A., \& Andersen, J. 2010, in Highlights of Spanish Astrophysics V, ed. J. M. Diego, L. Goicoechea, J. I. González-Serrano, \& J. Gorgas (Berlin: Springer-Verlag), 211

Dorren, J. D. 1987, ApJ, 320, 756

Fabrycky, D., \& Tremaine, S. 2007, ApJ, 669, 1298

Fabrycky, D. C., \& Winn, J. N. 2009, ApJ, 696, 1230

Ferraz-Mello, S., Tadeu Dos Santos, M., Beaugé, C., Michtchenko, T. A., \& Rodríguez, A. 2011, A\&A, 531, A161

Foukal, P. 1998, ApJ, 500, 958

Fulton, B. J., Howard, A. W., Winn, J. N., et al. 2013, ApJ, 772, 80

Gandolfi, D., Collier Cameron, A., Endl, M., et al. 2012, A\&A, 543, L5

Gaudi, B. S., \& Winn, J. N. 2007, ApJ, 655, 550

Gilliland, R. L., Jenkins, J. M., Borucki, W. J., et al. 2010, ApJL, 713, L160

Gizon, L., \& Solanki, S. K. 2003, ApJ, 589, 1009

Hatzes, A. P., Fridlund, M., Nachmani, G., et al. 2011, ApJ, 743, 75

Hirano, T., Sanchis-Ojeda, R., Takeda, Y., et al. 2012, ApJ, 756, 66

Hirano, T., Suto, Y., Winn, J. N., et al. 2011, ApJ, 742, 69

Holman, M. J., \& Murray, N. W. 2005, Sci, 307, 1288

Howell, S. B., Everett, M. E., Sherry, W., Horch, E., \& Ciardi, D. R. 2011, AJ, 142,19

Irwin, J., \& Bouvier, J. 2009, in IAU Symp. 258, The Ages of Stars, ed. E. E. Mamajek, D. R. Soderblom, \& R. F. G. Wyse (Cambridge: Cambridge Univ. Press), 363

Jenkins, J. M., Caldwell, D. A., Chandrasekaran, H., et al. 2010, ApJL, 713, L120

Koch, D. G., Borucki, W. J., Basri, G., et al. 2010, ApJL, 713, L79

Léger, A., Rouan, D., Schneider, J., et al. 2009, A\&A, 506, 287

Llama, J., Jardine, M., Mackay, D. H., \& Fares, R. 2012, MNRAS, 422, L72

Lovis, C., Dumusque, X., Santos, N. C., et al. 2011, arXiv:1107.5325

Mamajek, E. E., \& Hillenbrand, L. A. 2008, ApJ, 687, 1264

Mandel, K., \& Agol, E. 2002, ApJL, 580, L171

Meibom, S., Mathieu, R. D., \& Stassun, K. G. 2009, ApJ, 695, 679

Nesvorný, D., Kipping, D. M., Buchhave, L. A., et al. 2012, Sci, 336, 1133

Noyes, R. W., Hartmann, L. W., Baliunas, S. L., Duncan, D. K., \& Vaughan, A. H. 1984, ApJ, 279, 763
Nutzman, P. A., Fabrycky, D. C., \& Fortney, J. J. 2011, ApJL, 740, L10

Oshagh, M., Boisse, I., Boué, G., et al. 2013a, A\&A, 549, A35

Oshagh, M., Santos, N. C., Boisse, I., et al. 2013b, A\&A, 556, 19

Pál, A. 2008, MNRAS, 390, 281

Pont, F., Aigrain, S., \& Zucker, S. 2011, MNRAS, 411, 1953

Pont, F., Gilliland, R. L., Moutou, C., et al. 2007, A\&A, 476, 1347

Queloz, D., Bouchy, F., Moutou, C., et al. 2009, A\&A, 506, 303

Queloz, D., Eggenberger, A., Mayor, M., et al. 2000, A\&A, 359, L13

Rabus, M., Alonso, R., Belmonte, J. A., et al. 2009, A\&A, 494, 391

Rasio, F. A., \& Ford, E. B. 1996, Sci, 274, 954

Rogers, T. M., Lin, D. N. C., \& Lau, H. H. B. 2012, ApJL, 758, L6

Sanchis-Ojeda, R., Fabrycky, D. C., Winn, J. N., et al. 2012, Natur, 487, 449

Sanchis-Ojeda, R., \& Winn, J. N. 2011, ApJ, 743, 61

Sanchis-Ojeda, R., Winn, J. N., \& Fabrycky, D. C. 2013, AN, 334, 180

Sanchis-Ojeda, R., Winn, J. N., Holman, M. J., et al. 2011, ApJ, 733, 127

Scargle, J. D. 1982, ApJ, 263, 835

Schlaufman, K. C. 2010, ApJ, 719, 602

Schrijver, C. J., \& Title, A. M. 2001, ApJ, 551, 1099

Silva, A. V. R. 2003, ApJL, 585, L147

Silva-Valio, A. 2008, ApJL, 683, L179

Skumanich, A. 1972, ApJ, 171, 565

Smith, J. C., Stumpe, M. C., Van Cleve, J. E., et al. 2012, PASP, 124, 1000

Solanki, S. K. 2003, A\&ARv, 11, 153

Strassmeier, K. G., \& Rice, J. B. 1998, A\&A, 330, 685

Strassmeier, K. G., Rice, J. B., Wehlau, W. H., et al. 1991, A\&A, 247, 130

Stumpe, M. C., Smith, J. C., Van Cleve, J. E., et al. 2012, PASP, 124, 985

Torres, G., Fischer, D. A., Sozzetti, A., et al. 2012, ApJ, 757, 161

Tregloan-Reed, J., Southworth, J., \& Tappert, C. 2013, MNRAS, 428, 3671

Triaud, A. H. M. J. 2011, A\&A, 534, L6

Unruh, Y. C., \& Collier Cameron, A. 1995, MNRAS, 273, 1

Valenti, J. A., \& Fischer, D. A. 2005, ApJS, 159, 141

Vogt, S. S., Allen, S. L., Bigelow, B. C., et al. 1994, Proc. SPIE, 2198, 362

Winn, J. N. 2011, in Exoplanet Transits and Occultations, Exoplanets, ed. S. Seager (Tucson, AZ: Univ. of Arizona Press), 55

Winn, J. N., Fabrycky, D., Albrecht, S., \& Johnson, J. A. 2010a, ApJL, 718, L145

Winn, J. N., Johnson, J. A., Howard, A. W., et al. 2010b, ApJL, 723, L223

Winn, J. N., Noyes, R. W., Holman, M. J., et al. 2005, ApJ, 631, 1215

Yi, S. K., Demarque, P., Kim, Y.-C., et al. 2001, ApJS, 136, 417 